\title{
Geometric manipulation of the high-field linear magnetoresistance in InSb epilayers on GaAs (001)
}

\author{
W. R. Branford ${ }^{\text {a) }}$ \\ Blackett Laboratory, Imperial College, Prince Consort Rd., London, SW7 2BZ, United Kingdom, \\ and Department of Chemistry, UCL, Gordon Street, London WC1H OAJ, United Kingdom
}

\author{
A. Husmann \\ Cambridge Research Laboratory, Toshiba Research Europe Limited, 260 Cambridge Science Park, \\ Cambridge, United Kingdom \\ S. A. Solin \\ Department of Physics and Center for Materials Innovation, Washington University in St. Louis, \\ 1 Brookings Drive, St. Louis, Missouri 63141 \\ S. K. Clowes, T. Zhang, Y. V. Bugoslavsky, and L. F. Cohen \\ Blackett Laboratory, Imperial College, Prince Consort Rd., London, SW7 2BZ, United Kingdom
}

(Received 9 December 2004; accepted 21 March 2005; published online 13 May 2005)

\begin{abstract}
We address the inherent high-field magnetoresistance (MR) of indium antimonide epilayers on GaAs (001), studying the modification of the MR when processed into a set of geometries. The changes produced by the geometries are quite subtle. The extraordinary MR geometry produces the highest low-field MR while the Corbino geometry produces the largest high-field magnetoresistance. We demonstrate that any material with an unsaturating linear intrinsic MR, will also have an unsaturating linear Corbino MR, and that the ideal material for linear MR sensors in conventional geometries would have a high mobility and a small, linear intrinsic MR. (C) 2005 American Institute of Physics. [DOI: 10.1063/1.1923755]
\end{abstract}

The linear magnetoresistance (MR) of high mobility semiconductors, such as InSb, ${ }^{1} \mathrm{PbTe}^{2}$ and germanium ${ }^{3}$ has long been known and thin epilayers of these materials have been exploited ${ }^{4}$ for use in high-field sensors. In addition, interest in linear MR has been rekindled, first by the observation of the phenomenon in the silver chalcogenides ${ }^{5,6}$ and then in a range of ferromagnetic "dirty metals."7 A classical transport model of a uniform system cannot explain the nonsaturating linearity of the resistance in field and various mechanisms have been suggested. Broadly, the proposed models can be divided into three categories; (1) Sample inhomogeneities, ${ }^{8,9}$ (2) geometric boundary effects, ${ }^{10}$ and (3) quantum effects. ${ }^{11,12}$ Moreover, a geometric technique has recently been shown to massively enhance the low-field MR of extraordinary MR (EMR) ${ }^{13}$ hybrid structures, consisting of a metallic inclusion in a semiconductor matrix. In a related geometric model, Parish and Littlewood (PL) recently predicted $^{8}$ that the MR of an $(N \times M)$ square array of interconnected conducting regions in an insulating matrix would become linear and nonsaturating as the array becomes large $(N, M \geqslant 10)$. This is promising for high magnetic field sensor applications and was proposed ${ }^{8}$ as the mechanism of the large linear MR in the silver chalcogenides. Here, we report the use of lithographic techniques to fabricate disk arrays from a high mobility InSb thin film ${ }^{14}$ and compare the observed MR of these structures to the recent PL predictions, the intrinsic MR, the Corbino ${ }^{15} \mathrm{MR}$, and to an EMR device made from similar InSb material.

The various sample structures we address here are shown schematically in Fig. 1. van der Pauw (vdP), Corbino, EMR, and disk geometries fabricated from a $1.0 \mu \mathrm{m}$ thick InSb film, that was deposited on semi-insulating GaAs(100) substrates in a molecular-beam epitaxy system with base pressure of $10^{-10}$ mbar. ${ }^{14}$ The PL cloverleaf arrays, and the Corbino disk, were fabricated by optical lithography. The arrays consist of $100 \mu \mathrm{m}$ diameter circles, connected nominally by $6 \mu \mathrm{m}$ wide and $5 \mu \mathrm{m}$ long bridges. They were etched in 45:10:5 lactic acid:nitric acid:HF for $10 \mathrm{~s}$ at $30^{\circ} \mathrm{C}$. The resultant undercut reduced the bridge width to about $4 \mu \mathrm{m}$. Ti:Au was evaporated to provide Ohmic contacts to the disks. Sandblasting was used to shape the "as-grown" sample into a cloverleaf. The processing to produce the EMR device is described in detail elsewhere. ${ }^{16}$

Magnetotransport measurements were performed on (1 $\times 1),(9 \times 9)$, and $(10 \times 10)$ arrays, the Corbino disk and the as-grown film. The transverse and Hall MRs in magnetic fields of up to $8 \mathrm{~T}$ were measured at room temperature in a superconducting magnet, with the sample surface normal to

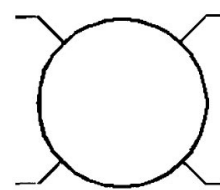

(a)

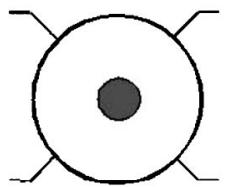

(c)

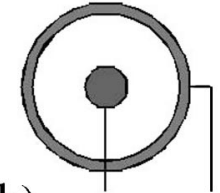

(b)

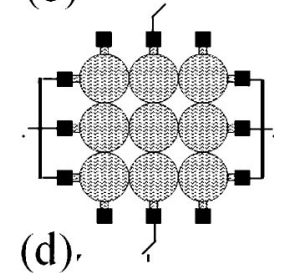

FIG. 1. Schematic diagrams of the sample geometries: (a) vdP, (b) Corbino, (c) EMR, and (d) disk array. The dark sections indicate metallic regions, in (d) the discs indicate InSb and the white background GaAs.

${ }^{a)}$ Electronic mail: w.branford@imperial.ac.uk 


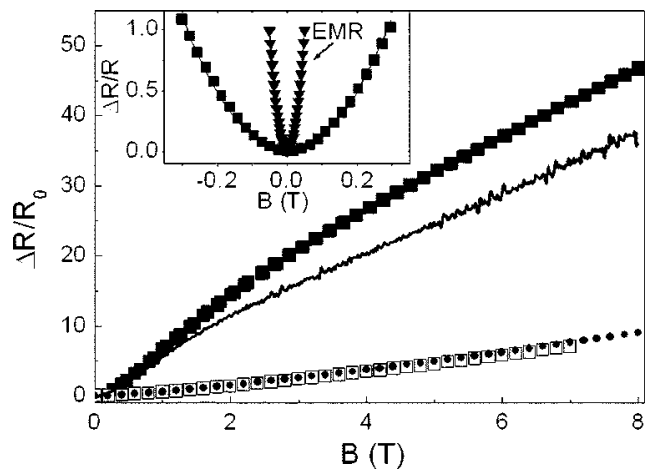

FIG. 2. Transverse $\left.\Delta R / R_{0}(\%)=100[R(B)-R(0 T)] / R(0 T)\right]$ of the as-grown film in the cloverleaf geometry (solid circles), the $1 \times 1$ processed array in the vdP geometry (open squares), and in the Corbino disk geometry (solid squares). The solid line shows the predicted Corbino MR if the measured $\mu_{H}(B)$ and $\Delta \rho(B) / \rho_{0}$ of the as-grown sample are inserted into Eq. (1). Inset: The low-field transverse MR of the Corbino disk (solid squares) and a fit to $\Delta R / R_{0}=\mu^{2} B^{2} ; \mu=3.5 \mathrm{~m}^{2} / \mathrm{V} \mathrm{s}$ (solid line), and the low-field MR of a macroscopic EMR device with circular metallic inclusion with filling factor 12/16th (taken from Ref. 16).

the applied magnetic field. For the $(9 \times 9)$ and $(10 \times 10)$ arrays, two current terminals were defined by electrically connecting all of the resistor units along two opposing edges. For the samples with the current terminals across the full width, the sheet resistance was determined from the product of the measured resistance and the aspect ratio, which is unity for $N=M$. The vdP method was used for the as-grown sample and the $1 \times 1$ array, both of which had current point contacts. Some sample geometries led to a weak mixing of the Hall and MR components, which were separated by their opposite symmetries with respect to inversion of the magnetic field.

The as-grown film was found to be $n$ type with $n$ $=1.95 \times 10^{22} \mathrm{~m}^{-3}$ and a low-field Hall mobility of $\mu_{H}$ $=3.7 \mathrm{~m}^{2} / \mathrm{V} \mathrm{s}$ at $300 \mathrm{~K}$. The processed single disk gave $n$ $=2.01 \times 10^{22} \mathrm{~m}^{-3}$ and $\mu_{H}=3.8 \mathrm{~m}^{2} / \mathrm{V} \mathrm{s}$, which indicates that the processing is not significantly altering the film. The mobility of the dominant transport carriers was verified with measurements on the Corbino disk; the low-field MR of which is given by

$$
\left(\Delta R / R_{0}\right)_{C}=\mu_{H}^{2} B^{2} /\left[1+\left(\Delta \rho / \rho_{0}\right)\right],
$$

where $\left(\Delta R / R_{0}\right)_{C}$ is the Corbino $\mathrm{MR},\left(\Delta \rho / \rho_{0}\right)$ is the intrinsic $\mathrm{MR}$, and $\mu^{2} B^{2}$ is the geometric MR ${ }^{17} \mathrm{~A}$ least-squares fit of Eq. (1) to the measured low-field is shown in the inset of Fig. 2 and yields $\mu=3.5 \mathrm{~m}^{2} / \mathrm{V} \mathrm{s}$ in good agreement with the Hall result. Also shown in Fig. 2 are the transverse MR of the as-grown cloverleaf, the $(1 \times 1)$ disk, and the Corbino disk.

To test whether $\left(\Delta R / R_{0}\right)_{\mathrm{vdP}}=\left(\Delta \rho / \rho_{0}\right)$, we reproduce $\left(\Delta R / R_{0}\right)_{C}$ from Eq. (1), using only parameters measured on the as-grown vdP sample. In this material, the Hall mobility, $\mu_{H}=d\left(R_{H}\right) / R_{\square} d B$, where $R_{\square}$ is zero field sheet resistance, is a nontrivial function of field, and its measured dependence is plotted in the left hand inset to Fig. 3. The experimental curves of $\mu_{H}(B)$ and $\left(\Delta R / R_{0}\right)_{\mathrm{vdP}}(B)$ were combined as in Eq. (1), and the predicted $\mathrm{MR}_{C}$ is plotted as the dotted line in the main body of Fig. 2. The predicted line is in excellent agreement with the experimental data in low field, and in reasonable agreement in high field. Thus, $\left(\Delta R / R_{0}\right)_{\mathrm{vdP}}=\left(\Delta \rho / \rho_{0}\right)$ is a reasonable assumption.

The intrinsic MR of semiconductors can be described ${ }^{18}$ as a power series in $\mu B$ as a power series in $\mu B$
Downloaded 03 Jun 2009 to 131.227.178.130. Redistribution subject to AlP license or copyright; see http://apl.aip.org/apl/copyright.jsp

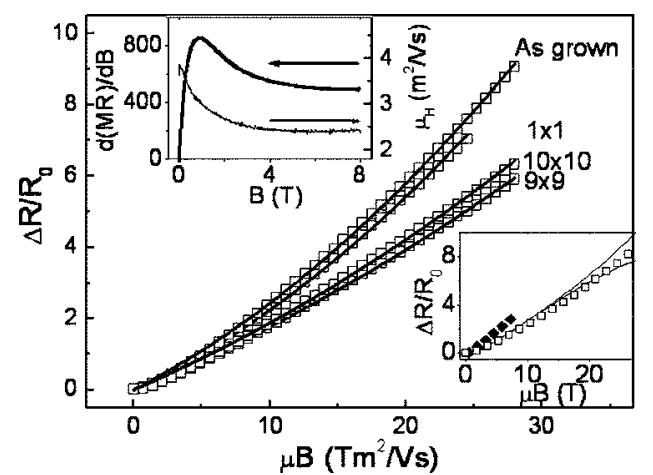

FIG. 3. Transverse MR $\left[\Delta R / R_{0}=[R(B)-R(0 T)] / R(0 T)\right]$ (open squares) of the as-grown film, and the $(1 \times 1),(9 \times 9)$, and $(10 \times 10)$ arrays, and fit of each data set to $\left[\Delta \rho / \rho_{0}=\alpha|\mu B|+\beta(\mu B)^{2}\right]$. The parameters obtained from this fitting procedure are shown in Table I. Inset left: Derivative $[d(\mathrm{MR}) / d B]$ of the MR of the Corbino disk (left axis) and Hall mobility $\left(\mu_{H}\right)$ (right axis) versus applied field. Inset right: MR of an unprocessed film (open squares) and the PL predictions for a material with $\mu=3.5 \mathrm{~m}^{2} / \mathrm{V} \mathrm{s}$ processed into a $9 \times 9$ (dotted line) and $10 \times 10$ (solid line) array. Also shown is the MR of the single disk at $1.5 \mathrm{~K}$ (solid diamonds).

$$
\Delta \rho / \rho_{0}=\alpha|\mu B|+\beta(\mu B)^{2} .
$$

This power series can be substituted into Eq. (1), giving the Corbino magnetorestance in

$$
\left(\Delta R / R_{0}\right)_{C}=\mu^{2} B^{2} /\left[1+\alpha|\mu B|+\beta(\mu B)^{2}\right] .
$$

The limits of Eq. (3) are very instructive for high-field MR sensor properties. If the sensor material has a linear intrinsic MR, i.e., $\alpha|\mu B| \gg 1, \beta(\mu B)^{2}$, then $\left(\Delta R / R_{0}\right)_{C}$ $=\mu B / \alpha$. If, on the other hand, there is a significant quadratic term in $\left(\Delta \rho / \rho_{0}\right)$, i.e., $\beta(\mu B)^{2} \gg \alpha|\mu B|, 1$, the MR will saturate and $\left(\Delta R / R_{0}\right)_{C}=1 / \beta$. For example, in our as-grown film $\alpha / \beta=41.7$, hence, the Corbino MR should saturate at $B$ $\gg 12 \mathrm{~T}$. Thus, for linear $\left(\Delta \rho / \rho_{0}\right),\left(\Delta R / R_{0}\right)_{C}$ will also be linear and unsaturating, with magnitude inversely proportional to that of $\left(\Delta \rho / \rho_{0}\right)$. Even in our InSb film, which has a very large $\left(\Delta \rho / \rho_{0}\right),\left(\Delta R / R_{0}\right)_{C}$ in the linear limit gives a massive enhancement to the intrinsic $\mathrm{MR}, d\left(\Delta R / R_{0}\right)_{C} / d B=4.9 / \mathrm{T}$, $\alpha \mu=0.69 \mathrm{~T}^{-1}$. The material we have studied is representative of micron-thick InSb epilayers on GaAs (001). (See Table I for a summary of InSb MR properties for samples from a range of sources.) Hence, the ideal material for a linear MR sensor in a standard geometry would have a large mobility and a small (though very linear) $\left(\Delta \rho / \rho_{0}\right)$.

In the vdP samples [cloverleaf and $(1 \times 1)$ disk], the $\left(\Delta \rho / \rho_{0}\right)$ should dominate. It can be seen from Fig. 2 that, although the vdP MR of the film is large, $\left(\Delta R / R_{0}\right)_{\mathrm{vdP}}(8 \mathrm{~T})$ $=885 \%$, it is dwarfed by $\left(\Delta R / R_{0}\right)_{C}(8 \mathrm{~T})=4685 \%$. Moreover, the low-field response of the Corbino device is greatly surpassed by the EMR device with filling factor 12/16th as shown in the inset of Fig. 2. Furthermore, neither $\left(\Delta R / R_{0}\right)_{C}$ nor $\left(\Delta R / R_{0}\right)_{\mathrm{vdP}}$ show any sign of saturating by $8 \mathrm{~T}$. In the case of $\left(\Delta R / R_{0}\right)_{C}$, this is better illustrated in the left inset of Fig. 3 by the derivative with respect to field $d\left(\Delta R / R_{0}\right)_{C} / d B$, which shows a maximum at the crossover from low-field to high-field dominated MR at $0.9 \mathrm{~T}$, and reaches a constant high-field limit above $6 \mathrm{~T}$ of $d\left(\Delta R / R_{0}\right)_{C} / d B=490 \% / \mathrm{T}$, indicating that $\left(\Delta R / R_{0}\right)_{C}$ is linear in $B$ between $6 \mathrm{~T}$ and $8 \mathrm{~T}$.

Consider now the MR of the disk arrays relative to the standard other geometries addressed here. If the bulk resis- 
TABLE I. Transport parameters obtained from the fitting of the room-temperature MR of the $1 \times 1,9 \times 9$, and $10 \times 10$ arrays to Eq. (1), as shown in Fig. 3. The same procedure has been applied to similar samples from Sheffield $\left(\mu=5.6 \mathrm{~m}^{2} / \mathrm{V} \mathrm{s}\right)$ and Emcore $\left(\mu=4.5 \mathrm{~m}^{2} / \mathrm{V} \mathrm{s}\right)$. For comparison, $1.5 \mathrm{~K}$ data $\left(\mu=0.9 \mathrm{~m}^{2} / \mathrm{V} \mathrm{s}\right)$ is also given for the single disk.

\begin{tabular}{lcccc}
\hline \hline \multicolumn{1}{c}{ Array } & $\begin{array}{c}\text { Sheet } \\
\text { Resistance } \\
(\Omega / \mathrm{cm})\end{array}$ & $\alpha$ & $\beta$ & $\alpha / \beta$ \\
\hline Unprocessed Imperial $(10 \mu \mathrm{m})$ & $86.8(1)$ & $0.196(1)$ & $0.0047(1)$ & $41.7(5)$ \\
Unprocessed Sheffield $(2.0 \mu \mathrm{m})$ & $23.8(1)$ & $0.131(1)$ & $0.0048(1)$ & $27.3(5)$ \\
Unprocessed Emcore $(1.3 \mu \mathrm{m})$ & $40.6(1)$ & $0.181(1)$ & $0.0043(1)$ & $42.1(5)$ \\
$1 \times 1$ array & $81.6(1)$ & $0.176(1)$ & $0.0047(1)$ & $37.4(5)$ \\
$9 \times 9$ array & $324.9(1)$ & $0.164(1)$ & $0.0023(1)$ & $71.3(5)$ \\
$10 \times 10$ array & $477.7(1)$ & $0.157(1)$ & $0.0020(1)$ & $78.5(5)$ \\
$1 \times 1$ array $(1.5 \mathrm{~K})$ & $843.0(1)$ & $0.284(1)$ & $0.0152(1)$ & $18.7(5)$ \\
\hline \hline
\end{tabular}

sheet resistance of an $(N \times N)$ square array would be independent of $N$. Note from Table I that the sheet resistance of the large arrays is nearly an order of magnitude larger than that of the vdP samples. This indicates that the zero-field resistance is dominated by the InSb bridges between disks, rather than the disks themselves. The transverse MR of the $(9 \times 9)$ and $(10 \times 10)$ arrays is compared to the vdP samples in Fig. 3. Clearly, the MR of our processed arrays is decreased relative to the vdP geometry, which is not surprising given the large extra term in the zero-field resistance from bottlenecking at the bridges. As a phenomenological test of how the disk structure influences the linear and quadratic MR terms, the MR of the $1 \times 1,9 \times 9$, and $10 \times 10$ arrays was fit to Eq. (2) so that the relative size of the linear and quadratic components can be compared.

The parameters $\alpha$ and $\beta$ obtained from this procedure are given in Table I. The ratio $\alpha / \beta$ gives an indication of the "linearity" of the MR. This shows that the $9 \times 9$ and 10 $\times 10$ arrays have a significantly increased linear component relative to the single disk. However, even in the single disk, the MR is much closer to linear than quadratic, and there is no evidence of saturation by $8 \mathrm{~T}$. Both the $9 \times 9$ and 10 $\times 10$ arrays are slightly superlinear. We note that the parameters $\alpha$ and $\beta$ of the single disk are greater at $1.5 \mathrm{~K}$ than at room temperature, although the MR (right hand inset to Fig. 3 ) is smaller because the mobility decreases to $0.9 \mathrm{~m}^{2} / \mathrm{V} \mathrm{s}$.

Calculations by Arora ${ }^{11}$ predicted linear MR for parabolic semiconductors in the high-field (quantum) $\operatorname{limit}^{19}$ and a slight superlinearity for a nonparabolic InSb conduction band. ${ }^{11}$ However, the similarity of the MR at $1.5 \mathrm{~K}$ and room temperature suggests that it is not a phonon-dominated process. Nevertheless, it is interesting to note that the PL model for large arrays describes the intrinsic linear MR behavior of the unprocessed InSb rather well, as is shown in the right hand inset to Fig. 3 particularly at room temperature. We stress that this represents generic InSb behavior and is not specific to our samples.

One of the initial aims of this work was to test the PL model. InSb was chosen because of its high mobility, and use as a commercial MR sensor. However, in the PL model, the bridges have negligible resistance, whereas our disks have high resistance interconnects. While our arrays do not perfectly reproduce the PL model, a comparison is still worthwhile.

The PL model predicts that for small arrays $(N<10)$, the MR will saturate in high field (be sublinear in $B$ ) when $N$ is odd and will not saturate (it will be superlinear in $B$ ) when $N$ is even. Moreover, for both odd and even $N$, the MR is predicted to become more linear as $N$ increases, and collapse onto the same straight line as $N$ tends to infinity (odd from above, and even from below). We do observe the increasing linearity with increasing $N$, which is of considerable interest if the disk structures can be exploited in the Corbino geometry. However, we do not observe either sublinearity for odd arrays or a boost in the MR over the vdP geometry, as predicted. The high resistance interconnects are the likely source of the deviations from the model predictions.

The authors thank M. Parish and P. Littlewood for data supplied and Sheffield growth facility for sample. The Imperial College portion of this work was funded by UK-EPSRC under Grant No. GR/R42402. One of the authors (W.R.B.) is supported by the Ramsay Memorial Fellowships Trust. Another author (S.A.S.) is supported by the U.S. National Science Foundation under Grant No. ECS-0329347.

${ }^{1}$ W. Schneider, H. Bruhms, and K. Hubner, J. Phys. Chem. Solids 41, 313 (1980).

${ }^{2}$ R. S. Allgaier, J. B. Restorff, and B. Houston, J. Appl. Phys. 53, 3110 (1982).

${ }^{3}$ J. W. Gallagher and W. F. Love, Phys. Rev. 161, 793 (1967).

${ }^{4} \mathrm{H}$. Weiss, Structure and Applications of Galvanomagnetic Devices (Pergamon, New York, 1969).

${ }^{5}$ R. Xu, A. Husmann, T. F. Rosenbaum, M. L. Saboungi, J. E. Enderby, and P. B. Littlewood, Nature (London) 390, 57 (1997).

${ }^{6}$ M. Lee, T. F. Rosenbaum, M. L. Saboungi, and H. S. Schnyders, Phys. Rev. Lett. 88, 066602 (2002).

${ }^{7}$ N. Manyala, Y. Sidis, J. F. DiTusa, G. Aeppli, D. P. Young, and Z. Fisk, Nature (London) 404, 581 (2000).

${ }^{8}$ M. M. Parish and P. B. Littlewood, Nature (London) 426, 162 (2003).

${ }^{9}$ C. Herring, J. Appl. Phys. 31, 1939 (1960).

${ }^{10}$ R. S. Allgaier, J. Appl. Phys. 59, 1388 (1986).

${ }^{11}$ V. K. Arora and M. Jaafarian, Phys. Rev. B 13, 4457 (1976).

${ }^{12}$ A. A. Abrikosov, Europhys. Lett. 49, 789 (2000).

${ }^{13}$ S. A. Solin, T. Thio, D. R. Hines, and J. J. Heremans, Science 289, 1530 (2000).

${ }^{14}$ T. Zhang, S. K. Clowes, M. Debnath, A. Bennett, C. Roberts, J. J. Harris, R. A. Stradling, L. F. Cohen, T. Lyford, and P. F. Fewster, Appl. Phys. Lett. 84, 4463 (2004).

${ }^{15}$ O. M. Corbino, Phys. Z. 12, 561 (1911).

${ }^{16}$ S. A. Solin, D. R. Hines, A. C. H. Rowe, J. S. Tsai, Y. A. Pashkin, S. J. Chung, N. Goel, and M. B. Santos, Appl. Phys. Lett. 80, 4012 (2002).

${ }^{17}$ K. Seeger, Semiconductor Physics (Springer, Wien-New York, 1973).

${ }^{18} \mathrm{H}$. Weiss, in Semiconductors and Semimetals Vol. 1, edited by R. K. Willardson and A. C. Beer (Academic, New York, 1966), p. 315.

${ }^{19}$ V. K. Arora and H. E. Spector, Phys. Status Solidi B 94, 323 (1979). 